\title{
Generalizing Krawtchouk Polynomials Using Hadamard Matrices
}

\author{
Peter S. Chami, ${ }^{1}$ Bernd Sing, ${ }^{1}$ and Norris Sookoo ${ }^{2}$ \\ ${ }^{1}$ Department of Computer Science, Mathematics and Physics, Faculty of Science and Technology, \\ The University of the West Indies, Cave Hill, St. Michael, Barbados \\ ${ }^{2}$ The University of Trinidad and Tobago, O'Meara Campus, Arima, Trinidad and Tobago \\ Correspondence should be addressed to Norris Sookoo; norris.sookoo@utt.edu.tt
}

Received 15 November 2013; Accepted 22 December 2013; Published 4 March 2014

Academic Editors: F. Ding and X. Liu

Copyright (C) 2014 Peter S. Chami et al. This is an open access article distributed under the Creative Commons Attribution License, which permits unrestricted use, distribution, and reproduction in any medium, provided the original work is properly cited.

We investigate polynomials, called $m$-polynomials, whose generator polynomial has coefficients that can be arranged in a square matrix; in particular, the case where this matrix is a Hadamard matrix is considered. Orthogonality relations and recurrence relations are established, and coefficients for the expansion of any polynomial in terms of $m$-polynomials are obtained. We conclude this paper by an implementation of $m$-polynomials and some of the results obtained for them in Mathematica.

\section{Introduction}

Matrices have been the subject of much study, and large bodies of results have been obtained about them. We study the interplay between the theory of matrices and the theory of orthogonal polynomials. For Krawtchouk polynomials, introduced in [1], interesting results have been obtained in [2-4]; also see the review article [5] and compare [6] for generalized Krawtchouk polynomials. More recently, conditions for the existence of integral zeros of binary Krawtchouk polynomials have been obtained in [7], while properties for generalized Krawtchouk polynomials can be found in [8]. Other generalizations of binary Krawtchouk polynomials have also been considered; for example, some properties of binary Krawtchouk polynomials have been generalised to $q$ Krawtchouk polynomials in [9]. Orthogonality relations for quantum and $q$-Krawtchouk polynomials have been derived in [10], and it has been shown that affine $q$-Krawtchouk polynomials are dual to quantum $q$-Krawtchouk polynomials. In this paper, we define and study generalizations of Krawtchouk polynomials, namely, $m$-polynomials.

The Krawtchouk polynomial $K_{k}(x)$ is given by

$$
K_{k}(x)=\sum_{j=0}^{k}(-1)^{j}\left(\begin{array}{c}
x \\
j
\end{array}\right)\left(\begin{array}{c}
n-x \\
k-j
\end{array}\right)
$$

where $n$ is a natural number and $x \in\{0,1, \ldots, n\}$. The generator polynomial is

$$
(1+z)^{n-x}(1-z)^{x}=\sum_{k=0}^{n} K_{k}(x) z^{k} .
$$

The generalized Krawtchouk polynomial $K_{p}(s)$ is obtained by generalizing the above generator polynomial as follows:

$$
\prod_{l=0}^{q-1}\left(\sum_{i=0}^{q-1} \chi\left(\bar{\omega}_{i} \bar{\omega}_{l}\right) z_{i}\right)^{s_{i}}=\sum_{p \in V(n, q)} K_{p}(s) z^{p},
$$

where $\sum s_{i}=\sum p_{i}=n, q$ is a prime power, the $z_{i}$ are indeterminate, the field $G F(q)$ with $q$ elements is $\left\{0, \bar{\omega}_{1}\right.$, $\left.\bar{\omega}_{2}, \ldots, \bar{\omega}_{q-1}\right\}$, and $\chi$ is a character.

The above information about Krawtchouk polynomials and generalized Krawtchouk polynomials was taken from [6].

If we replace the $\chi\left(\bar{\omega}_{i} \bar{\omega}_{l}\right)$ by arbitrary scalars in the last equation, we obtain the generator polynomial of $m$ polynomials $M G(p, s)$; see Definition 2 below. These $m$ polynomials are the subject of study in this paper.

In Section 2, we present relevant notations and definitions. In Section 3, we introduce the generator polynomial. The associated matrix of coefficients $G$ can be any square matrix, and so the question that immediately arises is how 
the properties of the $m$-polynomials are related to the properties of $G$. We will establish that, if $G$ is a generalized Hadamard matrix, then the associated $m$-polynomials satisfy orthogonality conditions. In Section 4, we establish recurrence relations for $m$-polynomials. Afterwards, we obtain coefficients for the expansion of a polynomial in terms of $\mathrm{m}$ polynomials in Section 5. Finally, in Section 6, we implement the results obtained here in Mathematica, so the reader may easily derive and explore $m$-polynomials for any matrix $G$.

\section{Definitions and Notations}

In this paper, $\mathbb{N}_{0}$ denotes $\{0,1,2,3, \ldots\}$. We use the convention that if $p \in \mathbb{N}_{0}^{q}$, then $p$ has components $\left(p_{0}, p_{1}, \ldots, p_{q-1}\right)$. So, if $r_{(i)} \in \mathbb{N}_{0}^{q}$ then $r_{(i)}$ denotes $\left(r_{i, 0}, r_{i, 1}, \ldots, r_{i, q-1}\right)$. We will also use the $q$ elementary unit vectors $e_{0}=(1,0,0, \ldots, 0)$, $e_{1}=(0,1,0, \ldots, 0), \ldots, e_{q-1}=(0,0, \ldots, 0,1)$.

We use the $\ell^{1}$-norm (the "taxicab-metric") to measure the length $|p|$ of $p \in \mathbb{N}_{0}^{q}$, that is, $|p|=\sum_{i=0}^{q-1} p_{i}$. We define the set of weak compositions of $n$ into $q$ numbers by $V(n, q)=$ $\left\{p \in \mathbb{N}_{0}^{q}:|p|=n\right\}$; in other words, $V(n, q)$ is the subset of $q$-dimensional nonnegative vectors of length $n$. We note that the set $V(n, q)$ has cardinality $\left(\begin{array}{c}n+q-1 \\ q-1\end{array}\right)$. For $p \in \mathbb{N}_{0}^{q}$, we use the multi-index notation $p !=\prod_{i=0}^{q-1} p_{i} !$. Similarly, for a variable $z=\left(z_{0}, z_{1}, \ldots, z_{q-1}\right) \in \mathbb{C}^{q}$ and $p \in \mathbb{N}_{0}^{q}$, we write $z^{p}=\prod_{j=0}^{q-1} z_{j}^{p_{i}}$ (where the convention $0^{0}=1$ is used). We note that the multinomial theorem reads

$$
\begin{aligned}
\left(\sum_{i=0}^{q-1} z_{i}\right)^{n} & =\sum_{p \in V(n, q)}\left(\begin{array}{l}
n \\
p
\end{array}\right) \cdot z^{p} \\
& =\sum_{p \in V(n, q)} \frac{n !}{p_{0} ! \cdots p_{q-1} !} \cdot z_{0}^{p_{0}} \cdots z_{q-1}^{p_{q-1}}
\end{aligned}
$$

In the following, $G$ denotes an arbitrary $q \times q$ matrix. We use the following convention to refer to the entries of a $q \times q$ matrix [11]. The entry in the $i$ th row and $j$ th column is called the $(i-1, j-1)$ entry, where $i, j=1,2, \ldots, q$. Thus, the $(i, j)$ entry of $G$ is denoted by $g_{i j}$, where $i, j=0,1, \ldots, q-1$. Given a matrix $G$, the matrix that remains when the first row and the first column of $G$ are removed is called the core of $G$.

The next definition is well known.

Definition 1. For an integer $q$ greater than one, a $q \times q$ matrix $H$ is called a generalized Hadamard matrix if $H \bar{H}^{T}=q I_{q}$, where $\bar{H}^{T}$ is the complex conjugate transpose of $H$ and $I_{q}$ is the $q \times q$ identity matrix. G.

We now define the $m$-polynomial with respect to a matrix

Definition 2. Let $s=\left(s_{0}, s_{1}, \ldots, s_{q-1}\right)$ and $p=\left(p_{0}, p_{1}, \ldots\right.$, $\left.p_{q-1}\right)$ be elements of $V(n, q)$. The m-polynomial in $s$ with respect to $G$ having parameter $p$ is denoted by $M G(p ; s)$ or $M G\left(p_{0}, p_{1}, \ldots, p_{q-1} ; s_{0}, s_{1}, \ldots, s_{q-1}\right)$ and given by

$$
\begin{aligned}
M G(p ; s) & =\sum_{r_{i, j}} \frac{s !}{\prod_{i, j} r_{i, j} !} \prod_{a=0}^{q-1} \prod_{b=0}^{q-1} g_{a b}^{r_{a, b}} \\
& =s ! \sum_{r_{i, j}} \prod_{a=0}^{q-1} \prod_{b=0}^{q-1} \frac{g_{a b}^{r_{a, b}}}{r_{a, b} !}
\end{aligned}
$$

where the summation is taken over all sets of nonnegative integers $r_{i, j}$ (with $i, j=0,1, \ldots, q-1$ ) satisfying

$$
\begin{aligned}
& \sum_{j=0}^{q-1} r_{i, j}=s_{i}, \quad i=0,1, \ldots, q-1, \\
& \sum_{i=0}^{q-1} r_{i, j}=p_{j}, \quad j=0,1, \ldots, q-1 .
\end{aligned}
$$

\section{The Generator Polynomial and Orthogonality Relations}

The values of the $m$-polynomial with respect to a matrix $G$ can be derived from a generator polynomial.

Theorem 3 (generator polynomial). Let $s=\left(s_{0}, s_{1}, \ldots, s_{q-1}\right) \in$ $V(n, q)$ and let $G=\left(g_{i j}\right)$ be a $q \times q$ matrix. Then,

$$
\prod_{i=1}^{q-1}\left(\sum_{j=0}^{q-1} g_{i j} z_{j}\right)^{s_{i}}=\sum_{p \in V(n, q)} M G(p ; s) z^{p},
$$

where $p=\left(p_{0}, p_{1}, \ldots, p_{q-1}\right)$.

Proof. This is an application of the multinomial theorem (recall that for $r_{(i)} \in \mathbb{N}_{0}^{q}$, we have $r_{(i)}=\left(r_{i, 0}, r_{i, 1}, \ldots, r_{i, q-1}\right)$ ):

$$
\begin{aligned}
\prod_{i=0}^{q-1}\left(\sum_{j=0}^{q-1} g_{i j} z_{j}\right)^{s_{i}}= & \prod_{i=0}^{q-1}\left\{\sum_{r_{(i)} \in V\left(s_{i}, q\right)} \frac{s_{i} !}{r_{(i)} !}\left[\prod_{j=0}^{q-1}\left(g_{i j} z_{j}\right)^{r_{i, j}}\right]\right\} \\
= & \sum_{\substack{r_{(i)} \in V\left(s_{i}, q\right), i=0,1, \ldots, q-1}} \frac{s !}{\prod_{i, j} r_{i, j} !}\left(\prod_{a=1}^{q-1} \prod_{b=0}^{q-1} g_{a b}^{r_{a, b}}\right) \\
& \times \prod_{k=0}^{q-1} z_{k}^{r_{0, k}+r_{1, k}+\cdots+r_{q-1, k}} \\
= & \sum_{p \in V(n, q)} M G(p ; s) z^{p}
\end{aligned}
$$

where $p_{k}=r_{0, k}+r_{1, k}+\cdots+r_{q-1, k}$.

As an immediate consequence, we can recover the entries $g_{i j}$ of the matrix $G$ as the $m$-polynomials of minimum order.

Corollary 4. We have $M G\left(e_{\ell} ; e_{k}\right)=g_{k \ell}$. 
Proof. For $s=e_{k}$, the left-hand side of (7) becomes

$$
g_{k, 0} z_{0}+g_{k, 1} z_{1}+\cdots+g_{k, q-1} z_{q-1},
$$

and since $p \in V(1, q)$ and thus runs through $e_{0}, \ldots, e_{q-1}$ in this case, the rest follows by comparing coefficients of $z_{j}$.

Remark 5. Theorem 3 can also be used for summation results of $m$-polynomials over $V(n, q)$ : using $z=(1, \ldots, 1)$, we obtain

$$
\sum_{p \in V(n, q)} M G(p ; s)=\prod_{i=0}^{q-1}\left(\sum_{j=0}^{q-1} g_{i j}\right)^{s_{i}}
$$

that is, the product of the $s_{i}$ th power of the $i$ th column sum of $G$.

As an immediate result of this remark, we can establish the following corollary.

Corollary 6. If $\sum_{j=0}^{q-1} g_{i j}=0$ for one $0 \leq i \leq q-1$ (i.e., one of the row-sums of the matrix $G$ is zero), then $\sum_{p \in V(n, q)} M G(p ; s)=0$.

For a generalized Hadamard matrix $G$, the multinomial theorem yields the following orthogonality relation for the corresponding $m$-polynomials.

Theorem 7 (orthogonality relation). If $G$ is a generalized Hadamard matrix, then the m-polynomials $\operatorname{MG}(p, s)$, with $p, s \in V(n, q)$, satisfy the orthogonality relations

$$
\sum_{s \in V(n, q)} \frac{1}{s !} M G(p ; s) \overline{M G(t ; s)}=\frac{q^{n}}{p !} \delta_{p, t},
$$

where

$$
\begin{gathered}
s=\left(s_{0}, s_{1}, \ldots, s_{q-1}\right), \quad p=\left(p_{0}, p_{1}, \ldots, p_{q-1}\right), \\
t=\left(t_{0}, t_{1}, \ldots, t_{q-1}\right), \\
\delta_{p, t}=\prod_{i=0}^{q-1} \delta_{p_{i}, t_{i}}= \begin{cases}1, & \text { if } p_{i}=t_{i} \\
0, & \text { otherwise }\end{cases}
\end{gathered}
$$

denotes Kronecker's delta.

Proof. Let $z=\left(z_{0}, z_{1}, \ldots, z_{q-1}\right), y=\left(y_{0}, y_{1}, \ldots, y_{q-1}\right) \in \mathbb{C}^{q}$, and define

$$
J=\sum_{i=0}^{q-1}\left(\sum_{j=0}^{q-1} g_{i j} z_{j}\right)\left(\sum_{k=0}^{q-1} \overline{g_{i k}} y_{k}\right)=\sum_{i=0}^{q-1} \sum_{j=0}^{q-1} \sum_{k=0}^{q-1} g_{i j} \overline{g_{i k}} z_{j} y_{k} .
$$

Then, on the one hand, we have

$$
J=q \sum_{j=0}^{q-1} z_{j} y_{j}
$$

since $G$ is a generalized Hadamard matrix. Consequently,

$$
J^{n}=q^{n}\left\{\sum_{s \in V(n, q)} \frac{n !}{s !}\left(z_{0} y_{0}\right)^{s_{0}}\left(z_{1} y_{1}\right)^{s_{1}} \cdots\left(z_{q-1} y_{q-1}\right)^{s_{q-1}}\right\} .
$$

On the other hand, the multinomial theorem yields

$$
\begin{aligned}
J^{n}= & \sum_{s \in V(n, q)} \frac{n !}{s !} \prod_{i=0}^{q-1}\left(\sum_{j=0}^{q-1} g_{i j} z_{j}\right)^{s_{i}}\left(\sum_{k=0}^{q-1} \overline{g_{i k}} y_{k}\right)^{s_{i}} \\
= & \sum_{s \in V(n, q)} \frac{n !}{s !}\left[\sum_{p \in V(n, q)} M G(p ; s) z^{p}\right] \\
& \times\left[\sum_{t \in V(n, q)} \overline{M G(t ; s)} y^{t}\right] .
\end{aligned}
$$

Equating coefficients of $z^{p} y^{t}$ in the two above expressions for $J^{n}$, we obtain the desired result.

If $G$ is a generalized Hadamard matrix and also satisfies certain additional conditions, then it is possible to establish that the corresponding $m$-polynomials satisfy additional orthogonality conditions. We use the following three results in proving this.

Lemma 8. If $G$ is symmetric, that is, $G=G^{T}$, then $M G(s ; p)=$ $(p ! / s !) M G(p ; s)$.

Proof. By Definition 2, we have (where $\sum_{j=0}^{q-1} h_{i, j}=p_{i}$ and $\left.\sum_{i=0}^{q-1} h_{i, j}=s_{j}\right)$

$$
\begin{aligned}
M G(s ; p) & =p ! \sum_{h_{i, j}} \prod_{a=0}^{q-1} \prod_{b=0}^{q-1} \frac{g_{a b}^{h_{a, b}}}{h_{a, b} !} \\
& =\frac{p !}{s !} s ! \sum_{h_{i, j}}^{q-1} \prod_{a=0}^{q-1} \frac{g_{b a}^{h_{a, b}}}{h_{a, b} !} \\
& =\frac{p !}{s !} s ! \sum_{r_{i, j}} \prod_{b=0}^{q-1} \prod_{a=0}^{q-1} \frac{g_{b a}^{r_{b, a}}}{r_{b, a} !} \\
& =\frac{p !}{s !} M G(p ; s),
\end{aligned}
$$

where we define $r_{i, j}=h_{j, i}$ (and thus $\sum_{j=0}^{q-1} r_{i, j}=s_{i}$ and $\sum_{i=0}^{q-1} r_{i, j}=p_{j}$ ), and since $G$ is symmetric (i.e., $g_{a b}=g_{b a}$ ).

The following results can be obtained in a similar manner to Lemma 8.

Lemma 9. If $G$ has a symmetric core, $g_{0, j}=1$ for $j=$ $0, \ldots, q-1$ and $g_{i, 0}=-1$ for $i=1, \ldots, q-1$, then $M G(s ; p)=$ $(-1)^{s_{0}+p_{0}}(p ! / s !) M G(p ; s)$.

Such symmetry relations yield additional orthogonality relations.

Theorem 10 (additional orthogonality relation). Let $G$ be a generalized Hadamard matrix. 
(i) If in addition $G$ is symmetric, then

$$
\sum_{s \in V(n, q)} M G(p ; s) \overline{M G(s ; t)}=q^{n} \delta_{p, t} .
$$

(ii) If in addition $G$ has a symmetric core, $g_{0, j}=1$ for $j=$ $0, \ldots, q-1$ and $g_{i, 0}=-1$ for $i=1, \ldots, q-1$, then

$$
\sum_{s \in V(n, q)}(-1)^{s_{0}} M G(p ; s) \overline{M G(s ; t)}=(-1)^{t_{0}} q^{n} \delta_{p, t} .
$$

Proof. This follows immediately from Theorem 7 and Lemmas 8 and 9.

Remark 11. It turns out that a slight modification of the above $m$-polynomials has already been considered in $[12,13]$. For a matrix $G$ and $p, s \in V(n, q)$, [13, Equation (2.3)] defines polynomials $L_{p, s}(G)$ by $L_{p, s}(G)=(1 / s !) M G(p ; s)$ in our notation. For these polynomials, the following multiplication property is proved (see [13, Theorem 3.2]):

$$
L_{p, t}\left(G_{1} G_{2}\right)=\sum_{s \in V(n, q)} s ! L_{p, s}\left(G_{1}\right) L_{s, t}\left(G_{2}\right),
$$

for $q \times q$ matrices $G_{1}, G_{2}$. With $G_{1}=G, G_{2}=\bar{G}^{T}$ and thus $G_{1} G_{2}=G \bar{G}^{T}=q I_{q}$, Theorem 7 becomes a special case of this equation by noting that $L_{s, t}\left(G^{T}\right)=L_{t, s}(G)$ (see [13, Equation (4.5)]) and $L_{p, t}\left(q I_{q}\right)=\left(q^{n} / p !\right) \delta_{p, t}$ (using the notation of Definition 2, the polynomial on the left can only be nonzero if $r_{i, j}=0$ whenever $i \neq j$ and $p_{i}=r_{i, i}=t_{i}$ otherwise).

\section{Recurrence Relations and Summation of Certain Polynomials}

We use Theorem 3 to derive a recurrence relation for $m$ polynomials in $s$ having parameter $p$ where both $s, p \in$ $V(n, q)$ by a sum of $m$-polynomials where both $s, p \in V(n-$ $1, q)$.

Theorem 12 (recurrence relations I). Let $p, s \in V(n, q)$ with $s_{i}>0$ for one $i \in\{0, \ldots, q-1\}$. Then,

$$
M G(p ; s)=\sum_{\substack{k=0, p_{k}>0}}^{q-1} g_{i k} M G\left(p-e_{k} ; s-e_{i}\right)
$$

where the sum on the right is taken over all $k$ such that the kth component $p_{k}$ of $p \in V(n, q)$ is positive.
Proof. Using (7) twice, we obtain (if $s_{i}>0$ )

$$
\begin{aligned}
& \sum_{p \in V(n, q)} M G(p ; s) z^{p} \\
& =\prod_{i=1}^{q-1}\left(\sum_{j=0}^{q-1} g_{i j} z_{j}\right)^{s_{i}} \\
& =\left(\sum_{j=0}^{q-1} g_{i j} z_{j}\right)\left(\sum_{j=0}^{q-1} g_{0 j} z_{j}\right)^{s_{0}} \ldots\left(\sum_{j=0}^{q-1} g_{i-1, j} z_{j}\right)^{s_{i-1}}, \\
& \left(\sum_{j=0}^{q-1} g_{i j} z_{j}\right)^{s_{i}-1}\left(\sum_{j=0}^{q-1} g_{i+1, j} z_{j}\right)^{s_{i+1}} \ldots\left(\sum_{j=0}^{q-1} g_{q-1, j} z_{j}\right)^{s_{q-1}} \\
& =\left(\sum_{j=0}^{q-1} g_{i j} z_{j} \sum_{\tilde{p} \in V(n-1, q)} M G\left(\tilde{p} ; s-e_{i}\right) z^{\tilde{p}} .\right.
\end{aligned}
$$

Multiplying the right-hand side out and equating coefficients of $z^{p}$ establish the claim.

Theorem 13 (recurrence relations II). Let $p, s \in V(n, q)$ with $p_{k}>0$ for one $k \in\{0, \ldots, q-1\}$. Then,

$$
M G(p ; s)=\sum_{\substack{i=0, s_{i}>0}}^{q-1} \frac{s_{i}}{p_{k}} g_{i k} M G\left(p-e_{k} ; s-e_{i}\right),
$$

where the sum on the right is taken over all $i$ such that the ith component $s_{i}$ of $s \in V(n, q)$ is positive.

Proof. We note that we have

$$
\frac{\partial}{\partial z_{k}}\left(\sum_{j=0}^{q-1} g_{i j} z_{j}\right)^{s_{i}}= \begin{cases}s_{i} g_{i k}\left(\sum_{j=0}^{q-1} g_{i j} z_{j}\right)^{s_{i}-1}, & \text { if } s_{i}>0 \\ 0, & \text { if } s_{i}=0 .\end{cases}
$$

Thus, on the one hand, the derivative of the left-hand side of (7) with respect to $z_{k}$ is

$$
\sum_{\substack{m=0, s_{m}>0}}^{q-1}\left[s_{m} g_{m k}\left(\sum_{\ell=0}^{q-1} g_{m \ell} z_{\ell}\right)^{s_{m}-1} \prod_{\substack{i=0, i \neq m}}^{q-1}\left(\sum_{j=0}^{q-1} g_{i j} z_{j}\right)^{s_{i}}\right] .
$$

Using (7), this is equal to

$$
\sum_{\substack{m=0, s_{m}>0}}^{q-1}\left[s_{m} g_{m k} \sum_{\tilde{p} \in V(n-1, q)} M G\left(\tilde{p} ; s-e_{m}\right) z^{\tilde{p}}\right] .
$$

On the other hand, the derivative of the right-hand side of (7) with respect to $z_{k}$ is

$$
\sum_{\substack{p \in V(n, q), p_{k}>0}} p_{k} M G(p ; s) z^{p-e_{k}}
$$


Equating the last two expressions and interchanging the order of summation yield

$$
\begin{gathered}
\sum_{\tilde{p} \in V(n-1, q)}\left[\sum_{\substack{m=0, s_{m}>0}}^{q-1} s_{m} g_{m k} M G\left(\tilde{p} ; s-e_{m}\right) z^{\tilde{p}}\right] \\
=\sum_{\substack{p \in V(n, q), p_{k}>0}} p_{k} M G(p ; s) z^{p-e_{k}} ;
\end{gathered}
$$

by comparing coefficients, we must have $\tilde{p}=p-e_{k}$, and therefore (noting that $p_{k}>0$ )

$$
M G(p ; s)=\sum_{\substack{m=0, s_{m}>0}}^{q-1} \frac{s_{m}}{p_{k}} g_{m k} M G\left(p-e_{k} ; s-e_{m}\right)
$$

We note that (21) and (23) can be rewritten as

$$
\begin{gathered}
M G(p ; s)=\sum_{\substack{r \in V(1, q), \text { s.t. } \\
p-r \in V(n-1, q)}} g_{i 0}^{r_{0}} g_{i 1}^{r_{1}} \cdots g_{i, q-1}^{r_{q-1}} \\
\times M G\left(p-r ; s-e_{i}\right), \\
M G(p ; s)=\sum_{\substack{r \in V(1, q), \text { s.t. } \\
s-r \in V(n-1, q)}} \frac{s_{0}^{r_{0}} s_{1}^{r_{1}} \cdots s_{q-1}^{r_{q-1}}}{p_{k}} g_{0 k}^{r_{0}} g_{1 k}^{r_{1}} \cdots g_{q-1, k}^{r_{q-1}} \\
\times M G\left(p-e_{k} ; s-r\right),
\end{gathered}
$$

respectively.

In particular, Theorem 12 can be easily iterated to obtain the following statements.

Proposition 14. Let $p, s \in V(n, q)$ with $s_{i} \geq m \geq 0$ for one $i \in\{0, \ldots, q-1\}$. Then,

$$
\begin{aligned}
M G(p ; s)= & \sum_{\substack{r \in V(m, q), \text { s.t. } \\
p-r \in V(n-m, q)}}\left(\begin{array}{c}
m \\
r
\end{array}\right) g_{i 0}^{r_{0}} g_{i 1}^{r_{1}} \cdots g_{i, q-1}^{r_{q-1}} \\
& \times M G\left(p-r ; s-m e_{i}\right),
\end{aligned}
$$

where the sum on the right is taken over all $r \in V(m, q)$ such that $p-r$ is in $V(n-m, q)$.

Proof. This follows by repeatedly applying (21) in Theorem 12. The multinomial coefficient $\left(\begin{array}{c}m \\ r\end{array}\right)$ arises as the number of ways $p$ can be changed to $p-r$ step-by-step.
Corollary 15. Let $p, s \in V(n, q)$ with $s_{i} \geq m_{i} \geq 0$ for all $i \in$ $\{0, \ldots, q-1\}$. Then,

$$
\begin{aligned}
M G(p ; s)= & \sum_{\substack{r_{(i)} \in V\left(m_{i}, q\right), s . t . \\
p-\sum_{i=0}^{q-1} r \in V(n-|m|, q)}}\left[\prod_{i=0}^{q-1}\left(\begin{array}{c}
m_{i} \\
r_{(i)}
\end{array}\right)\right] \\
& \times\left[\prod_{i=0}^{q-1} \prod_{j=0}^{q-1} g_{i j}^{r_{i j}}\right] M G\left(p-\sum_{i=0}^{q-1} r_{(i)} ; s-m\right),
\end{aligned}
$$

where the sum on the right is taken over all the $q-1$ vectors $r_{(i)} \in V\left(m_{i}, q\right)$ such that $p-\sum_{i=0}^{q-1} r \in V(n-|m|, q)$.

Proof. This follows from repeatedly applying Proposition 14, by subtracting $m_{0}$ from $s_{0}, m_{1}$ from $s_{1}$, and so on.

We note that taking $m=s$ in the previous corollary yields the statement of Definition 2 (since, by definition, $M G(0 ; 0)=$ $1)$.

Combining the recurrence relation in Theorem 12 with Theorem 7 yields the following summation formula for $m$ polynomials.

Proposition 16 (summation formula). Let $G$ be a generalized Hadamard matrix and $p, s \in V(n, q)$. Then, for any pair of fixed, arbitrary elements $i, j \in\{0, \ldots, q-1\}$, one has

$$
\sum_{s \in V(n, q)} \frac{1}{s !} M G\left(p+e_{j} ; s+e_{i}\right) \overline{M G(p ; s)}=g_{i j} \frac{q^{n}}{p !} .
$$

\section{Expansion of a Polynomial}

The coefficients for the expansion of a polynomial in terms of Krawtchouk polynomials have been obtained (cf. [6]). We obtain a similar result for $m$-polynomials defined in terms of a generalized Hadamard matrix, using orthogonality properties.

Theorem 17 (polynomial expansion). Let $G$ be a generalized Hadamard matrix, and let $x=\left(x_{0}, x_{1}, \ldots, x_{q-1}\right)$ be a variable element of $V(n, q)$.

(i) If $G$ is symmetric and the expansion of a polynomial $\gamma(x)$ in terms of m-polynomials defined with respect to $G$ is (with coefficients $\alpha_{s} \in \mathbb{C}$ )

$$
\gamma(x)=\sum_{s \in V(n, q)} \alpha_{s} \cdot M G(x ; s)
$$

then, for all $\ell \in V(n, q)$,

$$
\alpha_{\ell}=q^{-n} \sum_{i \in V(n, q)} \gamma(i) \cdot \overline{M G(\ell, i)} .
$$

Similarly, if the expansion of a polynomial $\gamma(x)$ in terms of m-polynomials is (with coefficients $\beta_{s} \in \mathbb{C}$ )

$$
\gamma(x)=\sum_{s \in V(n, q)} \beta_{s} \cdot M G(s ; x),
$$


then, for all $\ell \in V(n, q)$,

$$
\beta_{\ell}=q^{-n} \sum_{i \in V(n, q)} \gamma(i) \cdot \overline{M G(i, \ell)} .
$$

(ii) If $G$ has a symmetric core, $g_{0, j}=1$ for $j=0, \ldots, q-1$ and $g_{i, 0}=-1$ for $i=1, \ldots, q-1$, then the corresponding coefficients can be calculated for all $\ell \in V(n, q)$ by

$$
\alpha_{\ell}=(-1)^{\ell_{0}} q^{-n} \sum_{i \in V(n, q)}(-1)^{i_{0}} \gamma(i) \cdot \overline{M G(\ell, i)},
$$

respectively,

$$
\beta_{\ell}=(-1)^{\ell_{0}} q^{-n} \sum_{i \in V(n, q)}(-1)^{i_{0}} \gamma(i) \cdot \overline{M G(i, \ell)} .
$$

Proof. Let $x=i$. Multiplying both sides of (34) by $\overline{M G(\ell, i)}$ and summing each side over all elements of $V(n, q)$ yield

$$
\begin{aligned}
\sum_{i \in V(n, q)} \gamma(i) \overline{M G(\ell, i)} & =\sum_{i \in V(n, q)} \sum_{s \in V(n, q)} \alpha_{s} \overline{M G(\ell, i)} M G(i, s) \\
& =\sum_{s \in V(n, q)} \alpha_{s} \sum_{i \in V(n, q)} \overline{M G(l, i)} M G(i, s) \\
& =\sum_{s \in V(n, q)} \alpha_{s} \overline{\sum_{i \in V(n, q)} M G(l, i) \overline{M G(i, s)}} \\
& \stackrel{(\star)}{=} \sum_{s \in V(n, q)} \alpha_{s} \cdot q^{n} \cdot \delta_{\ell, s} \\
& =\alpha_{\ell} q^{n},
\end{aligned}
$$

where step $(\star)$ uses Theorem 10; the first result in (i) follows. The other results are proved similarly.

\section{Mathematica Code}

Here, we present Mathematica code to obtain $m$-polynomials. First, we specify the matrix $G$ and the length $n$ :

$$
\begin{aligned}
& >\mathrm{g}=\{\{1,1\},\{1,-1\}\} \\
& >\mathrm{n}=6
\end{aligned}
$$

From this we can calculate $q$, the set $V(n, q)$ and initialize the variable $z$ :

$$
\begin{aligned}
& >\mathrm{q}=\text { Union }[\operatorname{Dimensions}[\mathrm{g}]][[1]] \\
& >\text { pall = Sort }[\text { Flatten }[\text { Map [Permutations, } \\
& \text { IntegerPartitions }[\mathrm{n},\{\mathrm{q}\} \\
& \text { Range }[0, \mathrm{n}]]], 1]] \\
& >\mathrm{z}=\text { Table }[\mathrm{f}[\mathrm{i}],\{\mathrm{i}, \mathrm{q}\}]
\end{aligned}
$$

Using Theorem 3, we obtain the $m$-polynomial via its generator:

\footnotetext{
$>$ generator $\left[\mathrm{s}_{-}\right]:=$Expand $[$Product [

(Sum $[g[[i, j]] z[[j]]$,
}

$$
\begin{aligned}
& \left.\left.\{j, 1, q\}])^{-}(s[[i]]),\{i, 1, q\}\right]\right] \\
& >m g\left[p_{-}, s_{-}\right]:=\text {Coefficient [generator }[s], \\
& \text { Inner [Power, } z, p, \text { Times] }]
\end{aligned}
$$

The values of the $m$-polynomial can be shown using the following command:

$$
\begin{aligned}
& >\text { TableForm }[\text { Table }[\operatorname{mg}[\text { pall }[[i]], \\
& \text { pall }[[j]]],\{i, \text { Length }[\text { pall }]\}, \\
& \{j, \text { Length }[\text { pall }]\}], \text { TableHeadings }-> \\
& \{\text { pall, pall }\}, \text { TableDepth }->2]
\end{aligned}
$$

For the above chosen matrix $G$ and length $n$, the output for $M G(p ; s)$ looks as follows ( $p$ denotes the row and $s$ the column; e.g., $M G((2,4) ;(3,3))=3)$ :

\begin{tabular}{c|ccccccc} 
& $\{0,6\}$ & $\{1,5\}$ & $\{2,4\}$ & $\{3,3\}$ & $\{4,2\}$ & $\{5,1\}$ & $\{6,0\}$ \\
\hline$\{0,6\}$ & 1 & -1 & 1 & -1 & 1 & -1 & 1 \\
$\{1,5\}$ & -6 & 4 & -2 & 0 & 2 & -4 & 6 \\
$\{2,4\}$ & 15 & -5 & -1 & 3 & -1 & -5 & 15 \\
$\{3,3\}$ & -20 & 0 & 4 & 0 & -4 & 0 & 20 \\
$\{4,2\}$ & 15 & 5 & -1 & -3 & -1 & 5 & 15 \\
$\{5,1\}$ & -6 & -4 & -2 & 0 & 2 & 4 & 6 \\
$\{6,0\}$ & 1 & 1 & 1 & 1 & 1 & 1 & 1
\end{tabular}

Thus, we are able to evaluate the $m$-polynomial $M G(p ; s)$ for any $p, s \in V(n, q)$ (and thus check orthogonality and recurrence relations). With the help of the Mathematica function Fit, we can express the m-polynomials as univariant polynomials in $\left(s_{0}-s_{1}\right)$ as follows (we also recall that $\left|\left(s_{0}, s_{1}\right)\right|=s_{0}+s_{1}=6$ here $)$ :

$$
\begin{aligned}
M G\left((0,6) ;\left(s_{0}, s_{1}\right)\right)= & \frac{1}{720}\left(s_{0}-s_{1}\right)^{6}-\frac{5}{72}\left(s_{0}-s_{1}\right)^{4} \\
& +\frac{34}{45}\left(s_{0}-s_{1}\right)^{2}-1, \\
M G\left((1,5) ;\left(s_{0}, s_{1}\right)\right)= & \frac{1}{120}\left(s_{0}-s_{1}\right)^{5} \\
& -\frac{1}{3}\left(s_{0}-s_{1}\right)^{3}+\frac{11}{5}\left(s_{0}-s_{1}\right), \\
M G\left((2,4) ;\left(s_{0}, s_{1}\right)\right)= & \frac{1}{24}\left(s_{0}-s_{1}\right)^{4}-\frac{7}{6}\left(s_{0}-s_{1}\right)^{2}+3, \\
M G\left((3,3) ;\left(s_{0}, s_{1}\right)\right)= & \frac{1}{6}\left(s_{0}-s_{1}\right)^{3}-\frac{8}{3}\left(s_{0}-s_{1}\right), \\
M G\left((4,2) ;\left(s_{0}, s_{1}\right)\right)= & \frac{1}{2}\left(s_{0}-s_{1}\right)^{2}-3, \\
M G\left((5,1) ;\left(s_{0}, s_{1}\right)\right)= & \left(s_{0}-s_{1}\right), \\
M G\left((6,0) ;\left(s_{0}, s_{1}\right)\right)= & 1 .
\end{aligned}
$$


Similarly, we may express the $m$-polynomials as univariant polynomials in $\left(p_{0}-p_{1}\right)$ as follows:

$$
\begin{aligned}
& M G\left(\left(p_{0}, p_{1}\right) ;(0,6)\right)=\frac{77}{3840}\left(p_{0}-p_{1}\right)^{6}-\frac{203}{192}\left(p_{0}-p_{1}\right)^{4} \\
& +\frac{1519}{120}\left(p_{0}-p_{1}\right)^{2}-20 \\
& M G\left(\left(p_{0}, p_{1}\right) ;(1,5)\right)=\frac{7}{640}\left(p_{0}-p_{1}\right)^{5}-\frac{49}{96}\left(p_{0}-p_{1}\right)^{3} \\
& +\frac{131}{30}\left(p_{0}-p_{1}\right) \\
& M G\left(\left(p_{0}, p_{1}\right) ;(2,4)\right)=-\frac{7}{3840}\left(p_{0}-p_{1}\right)^{6}+\frac{7}{64}\left(p_{0}-p_{1}\right)^{4} \\
& -\frac{199}{120}\left(p_{0}-p_{1}\right)^{2}+4 \\
& M G\left(\left(p_{0}, p_{1}\right) ;(3,3)\right)=-\frac{7}{1920}\left(p_{0}-p_{1}\right)^{5} \\
& +\frac{19}{96}\left(p_{0}-p_{1}\right)^{3}-\frac{67}{30}\left(p_{0}-p_{1}\right), \\
& M G\left(\left(p_{0}, p_{1}\right) ;(4,2)\right)=\frac{7}{11520}\left(p_{0}-p_{1}\right)^{6}-\frac{25}{576}\left(p_{0}-p_{1}\right)^{4} \\
& +\frac{329}{360}\left(p_{0}-p_{1}\right)^{2}-4 \\
& M G\left(\left(p_{0}, p_{1}\right) ;(5,1)\right)=\frac{1}{384}\left(p_{0}-p_{1}\right)^{5} \\
& -\frac{17}{96}\left(p_{0}-p_{1}\right)^{3}+\frac{19}{6}\left(p_{0}-p_{1}\right) \\
& M G\left(\left(p_{0}, p_{1}\right) ;(6,0)\right)=-\frac{1}{2304}\left(p_{0}-p_{1}\right)^{6}+\frac{23}{576}\left(p_{0}-p_{1}\right)^{4} \\
& -\frac{101}{72}\left(p_{0}-p_{1}\right)^{2}+20 \text {. }
\end{aligned}
$$

By Theorem 17, we can express any polynomial $\gamma(x)=$ $\gamma\left(x_{0}, \ldots, x_{q-1}\right)$ in terms of $m$-polynomials. Using Mathematica to find the corresponding coefficients $\alpha_{s}$ and $\beta_{s}$ in the expansion in terms of $m$-polynomials, for example, for the polynomial $p\left(x_{0}, x_{1}\right)=x_{0} x_{1}$, is achieved as follows:

$$
\begin{aligned}
& >\mathrm{p}\left[\left\{\mathrm{x} 0_{-}, \mathrm{x} 1_{-}\right\}\right]:=\mathrm{x} 0 \mathrm{x} 1 \\
& >\text { Table[1/q } \text { Total }^{-} \text {Table[p[pall[[i]]] } \\
& \text { Conjugate }[\mathrm{mg}[\mathrm{pall}[[j]], \operatorname{pall}[[i]]]] \text {, } \\
& \{\text { i, Length }[\mathrm{pall}]\}]],\{j, \text { Length }[\mathrm{pall}]\}]
\end{aligned}
$$

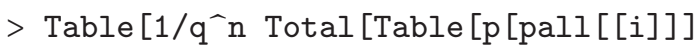

$$
\begin{aligned}
& \text { Conjugate }[\mathrm{mg}[\mathrm{pall}[[i]], \operatorname{pall}[[j]]]] \text {, } \\
& \{i, \text { Length }[\mathrm{pall}]\}]],\{j, \text { Length }[\mathrm{pall}]\}]
\end{aligned}
$$

The output of the later two lines is

$$
\left\{-\frac{3}{64}, 0,-\frac{39}{64}, 0, \frac{7}{64}, 0, \frac{35}{64}\right\}, \quad\left\{0,0,0,0,-\frac{1}{2}, 0, \frac{15}{2}\right\},
$$

giving the list of coefficients $\alpha_{s}$, respectively, $\beta_{s}$ for $s \in V(n, q)$. That is, the expansion in terms of $m$-polynomials here reads as follows:

$$
\begin{aligned}
x_{0} x_{1}= & -\frac{3}{64} \operatorname{MG}\left(\left(x_{0}, x_{1}\right) ;(0,6)\right) \\
& -\frac{39}{64} \operatorname{MG}\left(\left(x_{0}, x_{1}\right) ;(2,4)\right) \\
& +\frac{7}{64} \operatorname{MG}\left(\left(x_{0}, x_{1}\right) ;(4,2)\right) \\
& +\frac{35}{64} \operatorname{MG}\left(\left(x_{0}, x_{1}\right) ;(6,0)\right), \\
x_{0} x_{1}= & -\frac{1}{2} \operatorname{MG}\left((4,2) ;\left(x_{0}, x_{1}\right)\right) \\
& +\frac{15}{2} \operatorname{MG}\left((6,0) ;\left(x_{0}, x_{1}\right)\right) .
\end{aligned}
$$

As a check, we have (recall that $x_{0}+x_{1}=6$ )

$$
\begin{aligned}
-\frac{1}{2} & M G\left((4,2) ;\left(x_{0}, x_{1}\right)\right)+\frac{15}{2} M G\left((6,0) ;\left(x_{0}, x_{1}\right)\right) \\
& =-\frac{1}{2} \cdot\left(\frac{1}{2}\left(x_{0}-x_{1}\right)^{2}-3\right)+\frac{15}{2} \cdot 1 \\
& =x_{0}\left(6-x_{0}\right)=x_{0} x_{1} .
\end{aligned}
$$

For a generalized Hadamard matrix with symmetric core, $g_{0, j}=1$ for $j=0, \ldots, q-1$ and $g_{i, 0}=-1$ for $i=1, \ldots, q-1$; for example,

$$
G=\left(\begin{array}{ccc}
1 & 1 & 1 \\
-1 & \frac{1-\sqrt{3}}{2} & \frac{1+\sqrt{3}}{2} \\
-1 & \frac{1+\sqrt{3}}{2} & \frac{1-\sqrt{3}}{2}
\end{array}\right) .
$$

The above mathematica code using Theorem 17 has to be modified as follows:

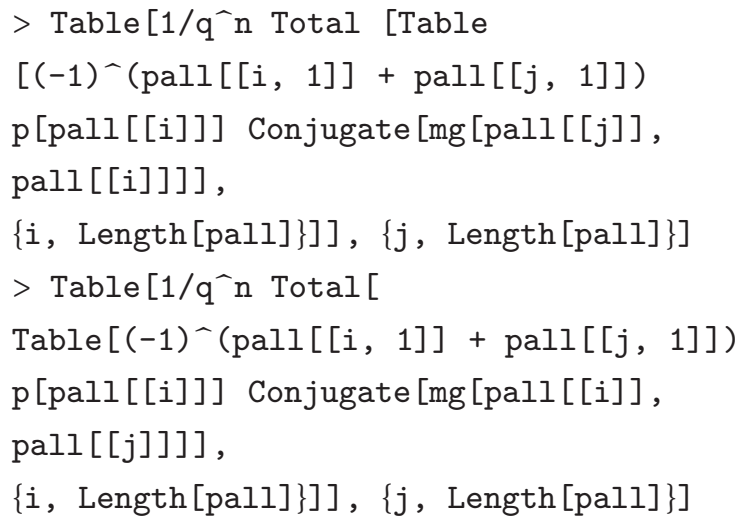

\section{Outlook}

Krawtchouk polynomials and their generalisation appear in many areas of Mathematics, see [5]: harmonic analysis $[1,2,4]$, statistics [3], combinatorics and coding theory 
$[6,7,9,11,14,15]$, probability theory [5], representation theory (e.g., of quantum groups) $[10,12,13]$, difference equations [16], and pattern recognition [17] (a website called the "Krawtchouk Polynomials Home Page" by V. Zelenkov at http://orthpol.narod.ru/eng/index.html collecting material about M. Krawtchouk and the polynomials that bear his name has, unfortunately, not been updated in a while). However, our motivation in this paper was primarily driven by generalising the results obtained in [8] —and thus shedding more light on the qualitative structure of (generalisations of) Krawtchouk polynomials - and not yet with a specific application in mind. By providing the Mathematica code to obtain $m$-polynomials, we also hope that other researchers are encouraged to explore them and see if they can be used in their research.

\section{Conflict of Interests}

The authors declare that there is no conflict of interests regarding the publication of this paper.

\section{References}

[1] M. Krawtchouk, "Sur une généralization des polynomes d'Hermite," Comptes Rendus de l'Académie des Sciences, vol. 189, pp. 620-622, 1929.

[2] G. Szego, Orthogonal Polynomials, vol. 23, Colloquium, New York, NY, USA, 1959.

[3] G. K. Eagleson, "A characterization theorem for positive definite sequences on the Krawtchouk polynomials," The Australian Journal of Statistics, vol. 11, pp. 29-38, 1969.

[4] C. F. Dunkl, "A Krawtchouk polynomial addition theorem and wreath products of symmetric groups," Indiana University Mathematics Journal, vol. 25, no. 4, pp. 335-358, 1976.

[5] P. Feinsilver and J. Kocik, "Krawtchouk polynomials and Krawtchouk matrices," in Recent Advances in Applied Probability, R. Baeza-Yates, J. Glaz, H. Gzyl, J. Hüsler, and J. L. Palacios, Eds., pp. 115-141, Springer, New York, NY, USA, 2005.

[6] F. J. MacWilliams, N. J. A. Sloane, and J.-M. Goethals, "The MacWilliams identities for nonlinear codes," The Bell System Technical Journal, vol. 51, pp. 803-819, 1972.

[7] I. Krasikov and S. Litsyn, "On integral zeros of Krawtchouk polynomials," Journal of Combinatorial Theory A, vol. 74, no. 1, pp. 71-99, 1996.

[8] N. Sookoo, "Generalized Krawtchouk polynomials: new properties," Archivum Mathematicum, vol. 36, no. 1, pp. 9-16, 2000.

[9] L. Habsieger, "Integer zeros of $q$-Krawtchouk polynomials in classical combinatorics," Advances in Applied Mathematics, vol. 27, no. 2-3, pp. 427-437, 2001.

[10] M. N. Atakishiyev and V. A. Groza, "The quantum algebra $U_{q}\left(s u_{2}\right)$ and $q$-Krawtchouk families of polynomials," Journal of Physics A, vol. 37, no. 7, pp. 2625-2635, 2004.

[11] P. Delsarte, "An algebraic approach to the association schemes of coding theory," Philips Research Reports, no. 10, 97 pages, 1973.

[12] J. D. Louck, "Group theory of harmonic oscillators in $n$ dimensional space," Journal of Mathematical Physics, vol. 6, pp. 1786-1804, 1965.

[13] W. Y. C. Chen and J. D. Louck, "The combinatorics of a class of representation functions," Advances in Mathematics, vol. 140, no. 2, pp. 207-236, 1998.
[14] V. I. Levenshtein, "Krawtchouk polynomials and universal bounds for codes and designs in Hamming spaces," IEEE Transactions on Information Theory, vol. 41, no. 5, pp. 1303-1321, 1995.

[15] F. J. MacWilliams and N. J. A. Sloane, Theory of Error-Correcting Codes, North-Holland, New York, NY, USA, 1978.

[16] L. Boelen, G. Filipuk, C. Smet, W. Van Assche, and L. Zhang, "The generalized Krawtchouk polynomials and the fifth Painleve equation," Journal of Difference Equations and Applications, vol. 19, pp. 1437-1451, 2013.

[17] J. Sheeba Rani and D. Devaraj, "Face recognition using Krawtchouk moment," Sädhanā, vol. 37, no. 4, pp. 441-460, 2012. 


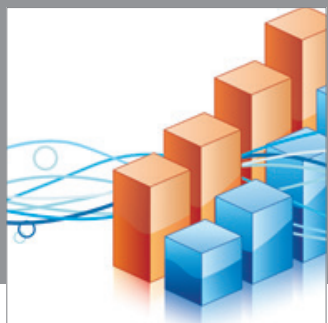

Advances in

Operations Research

mansans

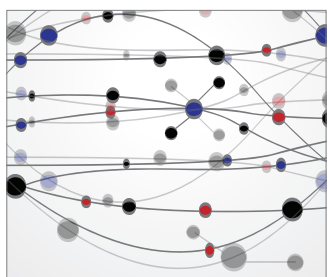

The Scientific World Journal
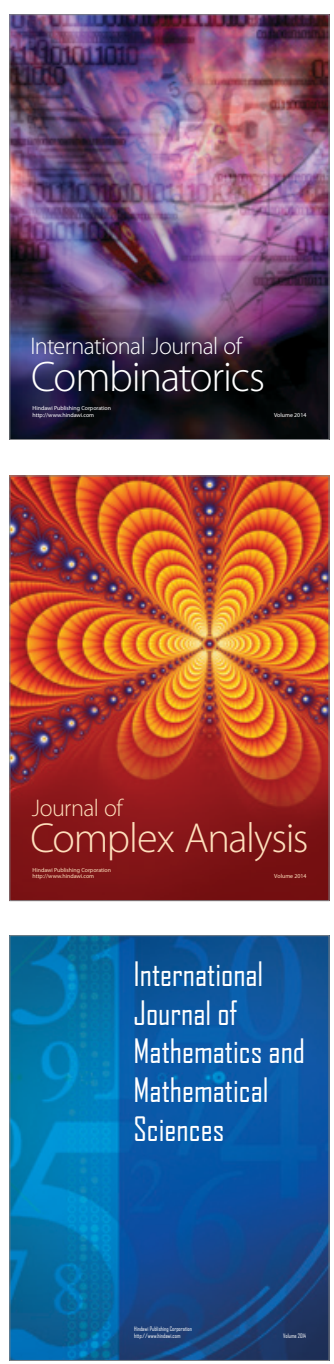
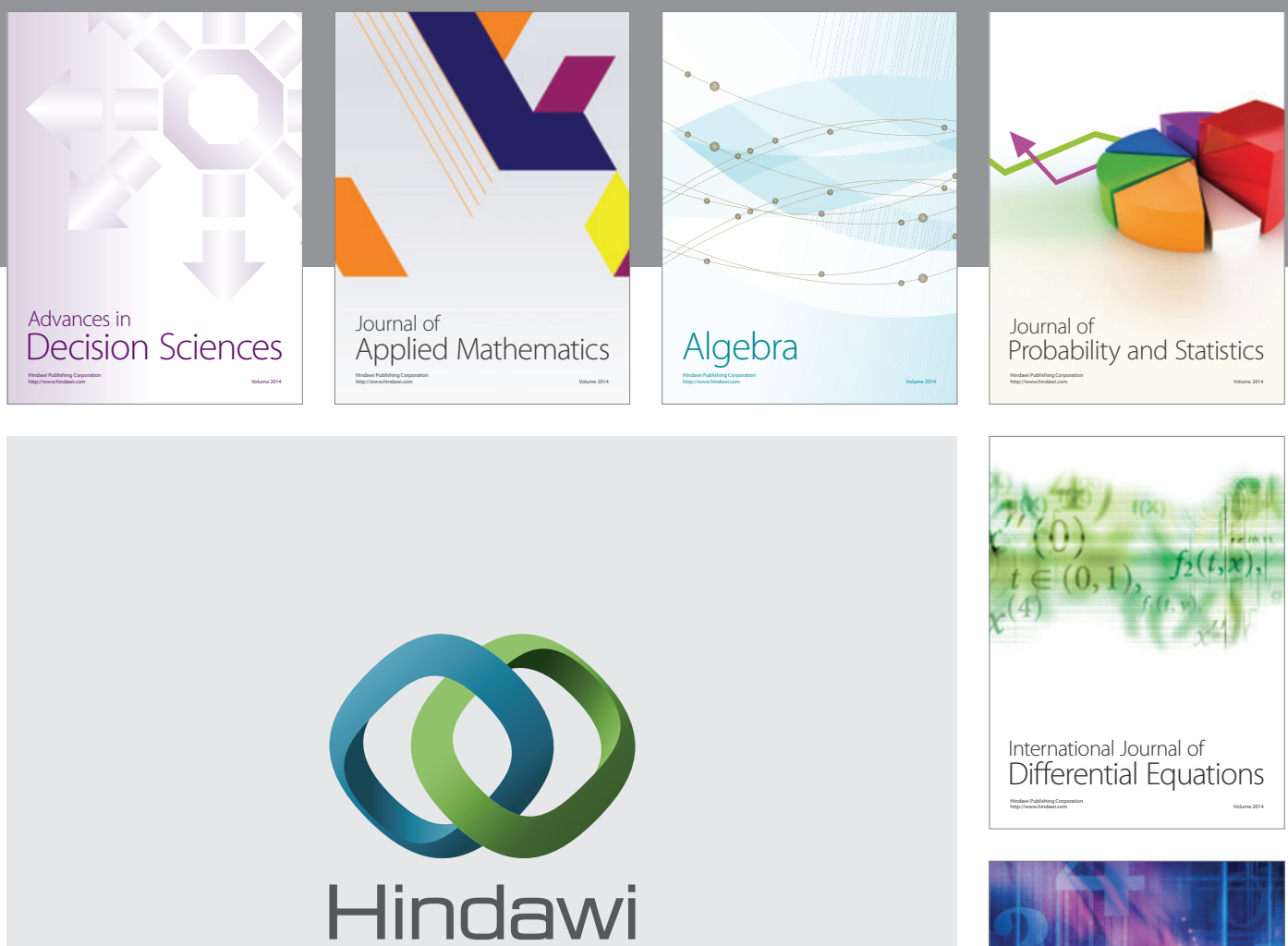

Submit your manuscripts at http://www.hindawi.com
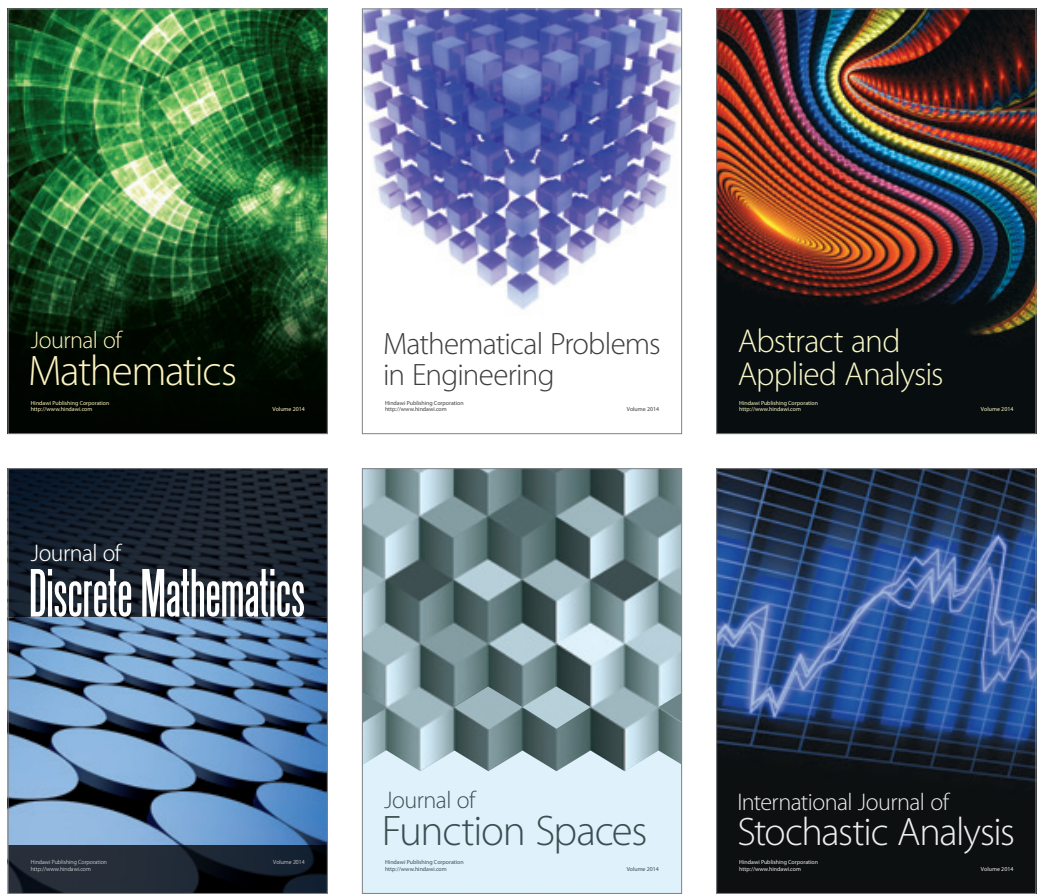

Journal of

Function Spaces

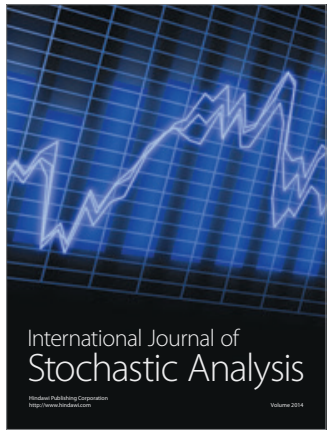

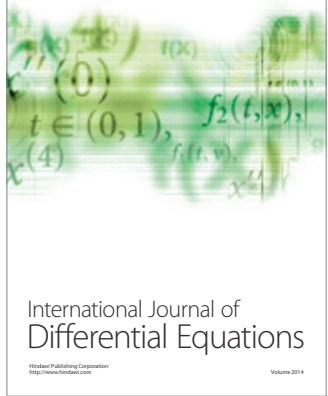
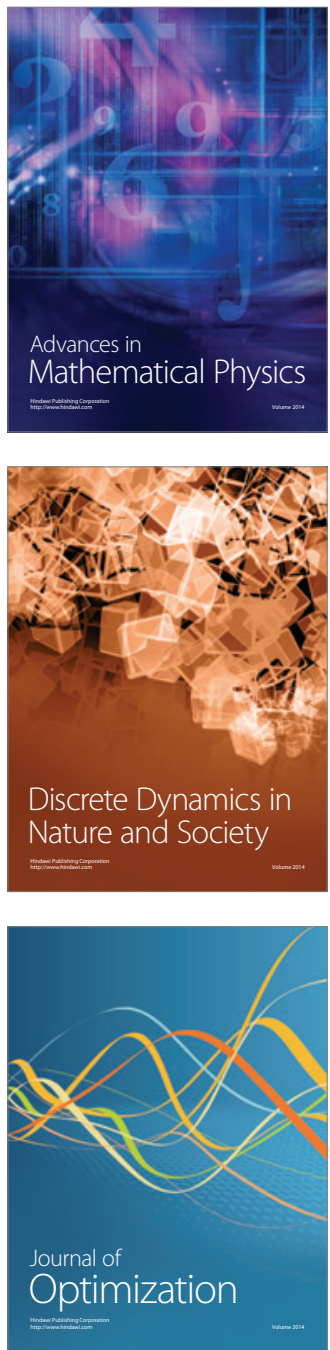